Review

\title{
CONTEMPORARY ASPECTS IN PATIENT HEALTH EDUCATION
}

\author{
P. Dzhedzheva* \\ Public Health Faculty, Specialty "Nurse", Affiliate Sliven, Medical University \\ "Prof. Dr. Paraskev Stoyanov", Varna, Bulgaria
}

\begin{abstract}
Achieving the highest possible level of health is one of the fundamental rights of every human being. In recent years, the demand for knowledge about understanding health and finding resources to achieve a higher quality of life has become increasingly relevant. Health education for patients and their families is an important component of the nurse's professional work.

The purpose of this paper is to present the results of an analysis of literature sources on contemporary aspects of health education for patients and their families.

Material and Methods: Sources and publications indexed in the following databases were used: Medline, EBSCO, Science Direct, Google Scholar, PubMed, Wiley online, as well as sites of the International Nursing Council and others.

Results: Based on the analysis of literature sources, we propose an organizational model for patient health education.

Conclusions: Health education needs to be prioritized and individualized. Involving the patient in the learning process will contribute to a more responsible attitude towards one's own health and greater independence.
\end{abstract}

Key words: health education, efficiency, patients, nurse, professional activity

\section{INTRODUCTION}

In 2012, all 53 Member States of the European Region of the World Health Organization (WHO) adopted a new common policy framework - Health 2020. They are united in the pursuit of the best possible health, regardless of ethnicity, gender, age, social status or solvency, as a fundamental right of every human being. This approach puts a new emphasis on measuring health and well-being instead of focusing on measuring disease, disability and mortality. This is in line with WHO's definition of health, as not just the absence of illness and disability, but as physical, social and mental well-being.

The demographic processes that have continued over several decades, such as population aging, reduced natural growth, and large emigration flows, have caused major

\footnotetext{
*Correspondence to: Pepa Dzhedzheva, $M U$ Varna, Affiliate Sliven, Public Health Faculty, Ul. General Stoletov 30, Sliven 8800, e-mail: p.djedjeva@gmail.com, mobile: +359887998985
}

changes in the age structure of the population, which are reflected in the demographic aging of the nation. Demographic aging significantly changes the structure of a nation's health needs and places certain demands on meeting them. Older people resort to healthcare more often, as illness, chronic illnesses, and hospital visits increase with age. The public is increasingly pressing for the pursuit of knowledge, understanding of health and finding the resources available in the healthcare system (1).

The healthcare system integrates activities that have proven to be effective, such as counselling in the field of a healthy lifestyle and preventive health care activities.

Overcoming the significant shortfall of knowledge, skills and motivation for healthy lifestyle among vulnerable groups and disadvantaged people will influence the causes of negative health trends. This requires active participation of the population (1). 
The nursing profession relates to the provision of care in the healthcare system. Patients are no longer passive recipients of medical services. Their quality requirements are high (2).

\section{PATIENT HEALTH EDUCATION}

Health education for patients and their families is an important component of the nurse's professional activities. Providing quality and effective education to patients will help to best meet their needs for supportive care, general and / or specific health care, residential care and social rehabilitation in an ambulatory or home setting and will significantly reduce the need from admission to hospitals for active treatment (1).

For many people, healthcare is an unfamiliar environment where they encounter unfamiliar vocabulary and concepts. Patients have the right to make informed decisions about their health. The information needed to make informed decisions must be accurate, complete and appropriate to the patient's needs.

Patient support improves the quality of life, health status, and satisfaction with health services, health awareness, and adherence to therapeutic activities (3).

Educating the patient is part of the treatment. The nurse guides the patient and their relatives on disease behaviour, health care, self-help, and treatment and rehabilitation assistance (4). Nurses provide patients with the information they need to take effective care of themselves after discharge from a hospital to ensure continuity of care, from hospital to home (5). Patients have the right to know and be informed about their diagnoses, prognosis and treatments available so that they can make adequate, informed decisions about their health and lifestyle. This will help them become healthier and more independent. Patientoriented educational approaches must be tailored to their health literacy and health culture. Creating a well-designed, comprehensive training plan that meets the unique needs of the patient, reduces health care costs, improves the quality of care, and ultimately alters behaviour to improve patient quality of life $(6,7,8)$.

The purpose of educating others about their health is to help people, families and communities achieve optimal levels of health.
DZHEDZHEVA $P$.

Patient education is an essential component of providing safe, patient-centred care (9).

Health education tailored to patients and their families aims to:

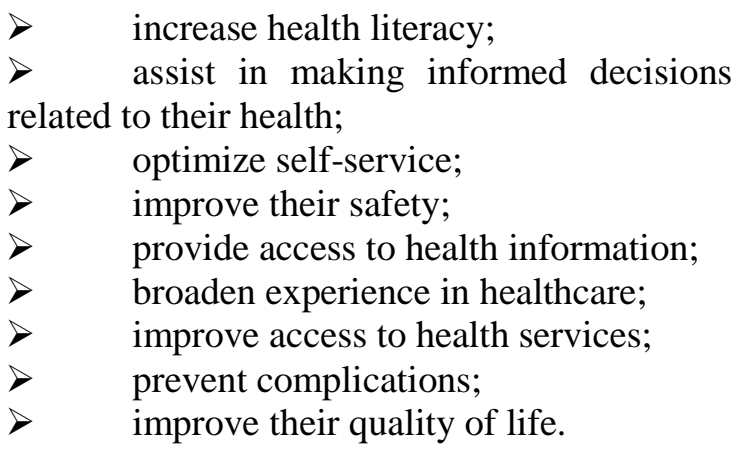

Health literacy refers to cognitive and social skills that determine people's motivation and ability to access health care, understand and use information in a way that promotes and maintains their good health (10).

Health literacy is fundamental to patient engagement. Unless people have the ability to receive, process and understand basic health information, they will not be able to take good care of themselves or make appropriate health decisions.

Health literacy is the ability to make adequate health decisions in the context of daily life - at home, in the community, at work, in the health care system, and more. This definition includes basic health knowledge; reading, understanding, and evaluating health information; health behaviour, etc. (11).

Inadequate health literacy can have profound health and financial consequences. Studies show that patients with low health literacy have a worse health status; are at greater risk of hospitalization; have less knowledge of disease management and health behaviour; do not actively participate in the health-related decision-making process, etc. (12).

Therefore, it is necessary to provide patients and their families with timely and appropriate health information materials that will enhance their health knowledge, skills and behaviours. This will enable them to make informed health decisions. Focusing on low literacy and disadvantaged groups will overcome inequalities in access to health care. Health literacy is crucial to raising the level of health culture of the population. 
Health culture, as part of public culture, is a system of knowledge, beliefs, values, habits, skills and behaviours to meet the need to protect, restore and promote personal and public health (13).

Health education is an influence on knowledge, perceptions, values, attitudes and behaviors related to health promotion (14).

Overall, patient education includes three important goals, each covering a separate phase of healthcare:

\section{Health promotion and disease prevention}

Health promotion and disease prevention are particularly important elements of public health.

Effective prevention is interdisciplinary prevention. It requires parallel action in several directions: improving the determinants of health (social, behavioral, environmental); involving the population in a healthy way of life; development and implementation of programs for the prevention and early diagnosis of chronic non-contagious diseases.

When people have the ability to control their health and the factors that determine it, communities become stronger and human life is better. Without the active participation of people, many opportunities for health promotion and protection, as well as for improving well-being, will be missed (1).

The nurse practitioner in primary care, hospital care, children's health care facilities, schools, etc. provides competent information and practical skills to children and adults for healthier behaviors to improve their physical and mental health. Promoting healthy behavior through education allows people to take greater responsibility for their own health (15). Greater knowledge leads to better habits for maintaining health. When patients become more aware of their health, they are more likely to seek early diagnosis of health problems (16)

\section{Restoration of health}

Patients with various illnesses need information and practical skills that will help them recover or maintain their desired level of health. During recovery and adaptation to changes resulting from illness, they are interested in information about their condition. Some patients find it difficult to adapt to the new state and become passive and uninterested in learning. The nurse should analyze patients' desire for training and motivate their interest in health education (17).

In order to achieve effective training for patients and their relatives, the nurse must analyze a number of factors: the patient's ability to cope with the disease, their conceptions of health, their socio-cultural level; the patient's needs, whether they are impaired or not.

Effective health education of patients starts from the moment of their hospitalization and continues until their discharge. Nurses working in hospital care should take every opportunity available throughout the patient's stay in the hospital to teach them self-care and selfcontrol skills.

For example, training patients how to inject insulin; in patients with colostomy - about colostomy bag replacement, hygiene, nutrition; for nursing mothers - about breastfeeding, hygiene care and bathing newborns, etc.

Without proper training, the patient can return to their home and resume unhealthy habits or ignore the management of their health. These actions can lead to relapse and rehospitalization.

The family also plays a major role in restoring patients' health. Family members who care for patients also need specific health training, including information on how to provide quality and safe care for their loved ones at home (18).

\section{Dealing with disturbed functions}

Not all patients fully recover from an illness. Many of them need to learn to cope with the constant changes in their health. In the event of serious impairment, patients and families need new knowledge and specific skills to accept changes in their physical abilities, to manage their lifestyles and to continue normal daily activities. After identifying the patient's needs, family members should be trained to assist them in managing their health status symptom monitoring, medication self-control, adequate physical activity and diet, psychosocial support, and more. Comparing the desired level of health with the actual state of health allows the planning of effective training programs. 
The education of patients and / or their relatives is a continuous two-way process, the effectiveness of which depends on the following conditions:

\section{Related to the nurse}

Level of professional qualification; knowledge, skills and competences to encourage individuals, families and groups of people to lead a healthy lifestyle and to take care of themselves, and to provide advice, guidance and support to persons in need of health care and their self-care relatives and others.

\section{Related to the patient}

The ability to receive, process and understand health information; state of health - in case of serious condition of the patient it is better to delay the training; intelligence; age and more.
DZHEDZHEVA $P$.

Certain conditions must be met for the education of patients and their family members to be effective:

$>$ a definite, clear purpose and motivation for acquiring knowledge;

$>\quad$ the nurse's benevolent attitude towards those being educated;

$>\quad$ creating an associative link between new information and past experience and knowledge;

$>$ mandatory practical applicability of new knowledge (19).

Based on the analysis of literature sources, we propose an organizational model for patient health education for the nurse (Figure 1), including the following activities:

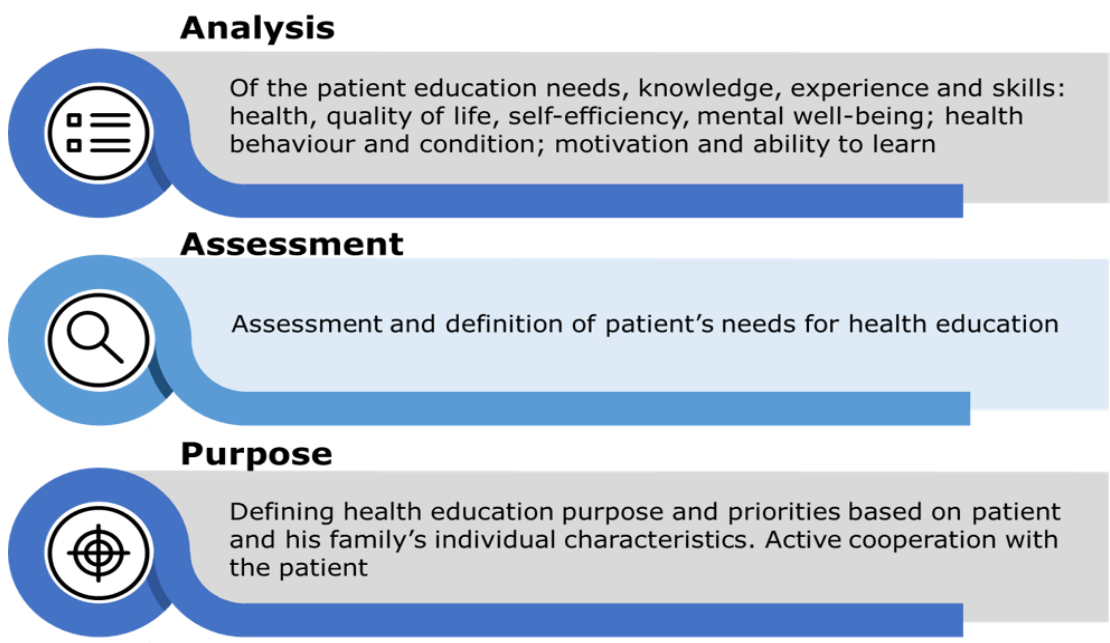

\section{Methods}

Defining the appropriate education methods, based on physical, mental and emotional limitations of the patient

\section{Application}

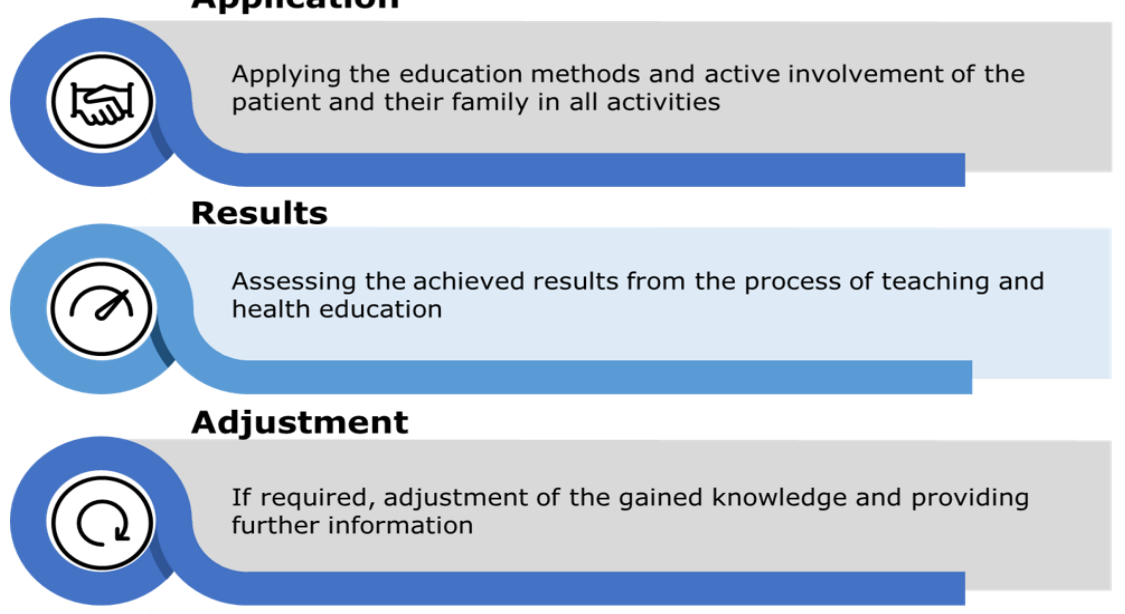

Figure 1. Organizational model for patient health education delivered by nurses 
1. Analysis of patients' needs for health education:

$>\quad$ Basic knowledge level, understanding of health status; possible complications; knowledge about self-care; knowing treatment options and possible outcomes; looking for and understanding health information;

$>\quad$ Patient's experience and knowledge: quality of life, self-efficiency; self-care activities; mental well-being;

$>\quad$ Health behaviours and health status: severity of the disease; activity levels; adherence to treatment; symptom control; family environment;

Motivation and ability to learn, etc.

2. Assessment and definition of patient's needs for health education;

3. Defining health education purpose and priorities based on patient and his family's individual characteristics. Active cooperation with the patient;

4. Defining the appropriate education methods, based on physical, mental and emotional limitations of the patient;

5. Defining the appropriate education methods, based on physical, mental and emotional limitations of the patient;

6. Assessing the achieved results from the process of teaching and health education;

7. If required, adjustment of the gained knowledge and providing further information (20).

\section{CONCLUSION}

Achieving effective health education for patients and their families will contribute to:

$>\quad$ Prevention of socially significant diseases such as obesity, diabetes, cardiovascular diseases, etc .;

$>\quad$ Patients' awareness of the disease: what changes to expect, recovery time, etc .;

$>\quad$ Reducing the risk of complications by educating patients and their family members about proper administration of medications, skills for handling a blood glucose meter or self-monitoring blood pressure monitor;

$>\quad$ Reducing hospital readmissions;

$>\quad$ Maintaining independence through self-care health training;

$>\quad$ Behavioral change to maintain health or maintain the maximum level of comfort;

$>\quad$ Improving the quality and longevity of patients with chronic diseases.

A proper educational approach that addresses individual needs, health literacy and active engagement will help patients have a more responsible attitude to their health and greater independence.

\section{REFERENCES}

1. Ministry of Health Bulgaria, Concept "Purposes for health 2020" Sofia, 2-9; 35; 53-54, 2015

2. http://www.mh.government.bg/media/filer_ public/2015/04/08/kontseptsiya-tseli-zazdrave-2020.pdf Accessed December 2019

3. Lebikyan E, P. Vasileva, Kompetenciite na medicinskite sestri $\mathrm{v}$ promeniashtoto se syvremenno obshtestvo, Nauchni trudove na Rusenskiq universitet, 48 seria, 8.1,150152, 2009

4. Vodenicharov, Tz., S. Popova, Socialna medicina, Sofia, 172-173, 2009

5. Toncheva, S., T. Tzvetkova, P. Zagorchev, S. Borisova, Narychnik za medicinski sestri $\mathrm{v}$ izvynbolnichna pomosht, pod redakciyata na K. Yurukova, Filvest, Sofia 2000

6. Falvo DR: Effective patient education: a guide to increased compliance, ed 4, Sudbury, Mass, Jones \& Bartlett, 2010

7. Edelman CL, Mandle CL: Health promotion throughout the life span, ed 7, St Louis, Mosby, 2010

8. Villablanca AC, et al: Outcomes of comprehensive heart care programs in highrisk women, J Women's Health 19(7): 1313, 2010.

9. Ministry of health Bulgaria, Zakon za zdraveto, chl. 81, 87, 88; 01.01.2005; izm i dop br.58, 23 July 2019

10.QSEN: Competency KSAs (pre-licensure), 2010,

http://www.qsen.org/ksas_prelicensure.php \#patient-centered_care. Accessed October 23, 2010.

11. Nutbeam D. Health literacy as a public health goal: a challenge for contemporary health education and communication strategies into the 21st century. Health Promot Int., 15(3):259-267, 2000

12.Kickbusch, I. Health Literacy: Addressing the health and education divide. - In: Health Promotion International,16(3):289297, 2001

13.Lennar Sihota, Saranjit \& Lennard, Linda. (2004). Health literacy: being able to make the most of health. 2004

14.Borisov, V., S. Popova, L. Georgieva, K. Shopova, Promociya na zdraveto.S., Arso, 108, 1998

15.Mladenova, S., Zdravno obrazovanie, Sofia, 21, 2009 
16.Longo DR, et al: Health information seeking, receipt, and use in diabetes selfmanagement, Ann Fam Med 8:334, doi: 10.1370/afm.1115, 2010,

17. Hawkins N, et al: Why the PAP test? Awareness and use of the PAP test among women in the United States, $J$ Womens Health 20(4):511, doi: 10.1089/ jwh2011.2730, 2011

18. Redman BK: The practice of patient education, ed 10, St Louis, Mosby, 2007
DZHEDZHEVA $P$.

19.Franciscan Missionaries of Our Lady University, The Role of Patient Education in

Nursing

https://online.franu.edu/news/2018/07/03/ro le-patient-education-nursing, July 2018. Accessed December 2019

20. Balkanska P., Za umenieto da obshtuvame s pacienta; sp. Sestrinsko delo; kn, 3, 26-30, 2009

21.Coulter A, Ellins J. Effectiveness of strategies for informing, educating, and involving patients. $B M J$. ,335:24-27, 2007 